\title{
A Case of Calvarial Tuberculosis in an Elderly Male Patient
}

\author{
Miqdad Haider ${ }^{1}$, Aijaz Zeeshan Khan Chachar², Nabeel Shafqat ${ }^{3}$, Waqar Zaheer ${ }^{4}$ \\ ${ }^{1}$ Registrar Department of Medicine, Fatima Memorial Hospital, Lahore Pakistan \\ ${ }^{2}$ Senior Registrar Department of Medicine, Fatima Memorial Hospital, Lahore Pakistan \\ ${ }^{3}$ Postgraduate Resident, DHQ Gujranwala Pakistan \\ ${ }^{4}$ Medical Officer, Social Security Hospital, Islamabad Pakistan
}

\begin{abstract}
A B S TR ACT
Tuberculosis (TB) is still endemic in developing countries like Pakistan. Tuberculosis affecting bones account for $1 \%$ of all TB infections. Calvarial tuberculosis, a rare manifestation of extra-pulmonary TB, accounts for $0.2 \%-1.3 \%$ of all cases of skeletal TB. Even in developing countries where TB is endemic, cases with this type of TB are not commonly seen. The most commonly involved sites are the frontal and parietal bones, with destruction of both the inner and outer table. Proper diagnosis and timely management help in improving prognosis. Here we describe the case of a 70-year-old male patient with pulmonary and extrapulmonary (calvarial) tuberculosis. The diagnosis was established on histopathological examination with demonstration of Acid-Fast Bacilli (AFB) and typical features seen on computed tomography (CT) scan.

Key Words: Calvarial TB, Pulmonary tuberculosis, Scalp swelling

$\begin{array}{ll}\text { Correspondence: } & \text { Article info: } \\ \text { Miqdad Haider } & \text { Received: October 21, 2020 } \\ \text { Email:miqdad14@yahoo.com } & \text { Accepted: October 27, 2020 }\end{array}$

Cite this case Report: Haider M, Chachar AZK, Shafqat N, Zaheer W. A Case of Calvarial Tuberculosis in an Elderly Male Patient. J Islamabad Med Dental Coll. 2021; 10(1): 62-65. Doi: 10.35787/jimdc.v10i1.624

Funding Source: Nil

Conflict of Interest: Nil

\section{Introduction}

Infection with Mycobacterium tuberculosis is still a common entity in our part of the world. There has been resurgence in even developed countries with increase in prevalence of Human Immunodeficiency Virus (HIV) infection. ${ }^{1}$ Mycobacterium can infect any organ of the body, although pulmonary manifestation is the most common one. ${ }^{2}$ Extrapulmonary involvement is seen in pericardium, meninges, kidneys, adrenal glands and bones, etc. ${ }^{3}$ Calvarial Tuberculosis involving the skull bones is a rare extra-pulmonary manifestation of TB. ${ }^{4}$

Approximately $1 \%$ of all patients with Tuberculosis ${ }^{1}$ have bone involvement and of these only $0.2 \%-1.3 \%$ are diagnosed as having TB of calvarium. ${ }^{2} \mathrm{~A}$ possible reason for this low incidence might be scarcity of lymphatics in the skull bone. Majority of the diagnosed patients are of young age (first two decades of life). ${ }^{5}$ The incidence of TB of skull although low at the moment, is on the rise in countries with poor socioeconomic status, prevalent malnutrition and also due to various immunodeficiency states.

Perforating tuberculosis of the skull is a term used by Volkmann ${ }^{6}$ to describe small punched-out lesions with granulation tissue covering both the inner and outer tables of the calvaria. Awasthy et al. ${ }^{6}$ described that it has low tendency to spread and is 
not linked with a periosteal reaction. These lesions are commonly known as circumscribed lytic lesions.

The most frequently seen clinical presentation of calvarial TB is a scalp swelling with discharge. Frontal and parietal bones are usually involved. ${ }^{5}$ Computed Tomography (CT) scan may show a lytic lesion and histopathological evidence is required for a definite diagnosis.

Our patient was a 70-year-old male who presented with a scalp swelling which was fluctuant on palpation.

\section{Case Report}

A 70-year-old male presented to the Outpatient Department (OPD) of Medicine, Fatima Memorial Hospital, Lahore, with complaints of cough for two months, fever (undocumented) since one month, shortness of breath on exertion and weight loss of about $5 \mathrm{~kg}$ over a period of three months. Patient was a non-smoker and belonged to lower income class. On examination, he was an old ill looking man, afebrile with blood pressure of $130 / 90 \mathrm{mmHg}$ and regular pulse of 84 beats/minutes. Chest auscultation revealed bronchial breath sounds in right upper lobe of the lung along with coarse crackles and decreased breath sounds in right lung base. Further examination revealed a painless swelling in the scalp, with no visible discharge. It was $3 \times 3 \mathrm{~cm}$ in diameter, non-tender, soft and fluctuant on palpation. He was admitted in the hospital for further workup and management.

Investigations showed hemoglobin level of 9.8 $\mathrm{mg} / \mathrm{dl}$, total leucocyte count $8500 / \mathrm{mm}^{3}$, differential leucocyte count with $60 \%$ polymorphs, $35 \%$ lymphocytes and erythrocyte sedimentation rate of $88 \mathrm{~mm} / \mathrm{hr}$. Serum creatinine level was $0.9 \mathrm{mg} / \mathrm{dl}$ and random blood sugar level was $136 \mathrm{mg} / \mathrm{dl}$. Urine examination showed 1-2 pus cells, and no glucose. Serum potassium ( $4.4 \mathrm{mmol} / \mathrm{L}$ ), serum sodium (139 $\mathrm{mmol} / \mathrm{L})$ and serum magnesium $(1.7 \mathrm{mg} / \mathrm{dL}$ ) were within the normal range. Chest $x$-ray revealed right upper lobe cavitating lesion and blunting of right costophrenic angles. High resolution computed tomography (HRCT) of chest confirmed findings of the chest $x$-ray, demonstrating wedge shaped cavity in right upper lobe with bilateral pleural effusions, confirmed by the consultant radiologist of the hospital. For the scalp swelling, $x$-ray of skull was done which showed erosion of the underlying bone. Necrotic debris was obtained on fine needle aspiration cytology (FNAC). Computed tomography (CT) scan was advised, which showed a subperiosteal abscess with punched out lytic lesion and extradural abscess (Figure 1). For a definitive diagnosis of this lesion, surgical intervention was performed. Tissue was excised, debridement done and the curettings were sent to the histopathology laboratory. The report revealed presence of caseous granulomas. Ziehl-Neelsen (ZN) staining of the sample showed Acid Fast Bacilli (AFB), hence confirming the diagnosis of tuberculosis of this region.

The patient was diagnosed as having pulmonary and extrapulmonary (calvarial) tuberculosis infection. Anti-tuberculous therapy (ATT) was started. ATT along with timely surgical intervention helped in improvement of the patient's condition. Patient was discharged after observation and was advised to follow up in the Medical OPD.

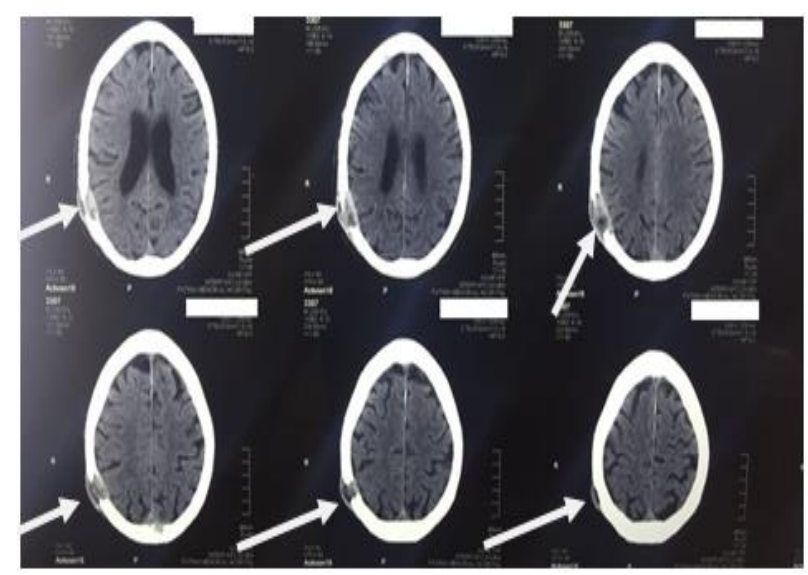

Figure 1: CT Brain plain showing punched-out lytic lesion (arrows) in the Parietal bone. 


\section{Discussion}

Calvarial tuberculosis is a very rare disease even in countries where TB is endemic. A primary source of TB (like pulmonary TB) may exist followed by spread through the hematogenous route. ${ }^{3,7}$ Calvarial TB as a single diagnosis is seen very rarely. About $75-90 \%$ patients with calvarial TB are under 20 years of age. 2,5

It has been observed to affect both sexes equally with no sex predilection. Early in the course of the disease the diagnosis can be easily missed because of lack of typical clinical signs. The appearance of a painless fluctuant scalp swelling is the most common presentation and usually the first symptom, as was seen in our patient. ${ }^{3,7}$ The scalp swelling is usually associated with erosion of the underlying bone. ${ }^{7}$ The most common sites for calvarial TB are the frontal and parietal bones and rarely in the occipital bone. ${ }^{5}$ Our patient also had involvement of the parietal bone of the skull.

The clinical findings depicting intracranial involvement such as seizures, meningitis and venous sinus thrombosis is rarely observed in calvarial TB. Our patient presented with scalp swelling with no discharge and pulmonary manifestations of TB but no evidence of neurological findings.

Mantoux test and raised erythrocyte sedimentation rate (ESR) are good markers for the diagnosis of TB along with other confirmatory tests. Mantoux test may be negative in $10 \%$ TB patients, while the ESR may be normal in $10 \%$ of patients. ${ }^{8}$

Findings of calvarial TB on $\mathrm{x}$-ray skull are variable and nonspecific, as seen in our patient who showed some erosion of the underlying bone. X-ray findings can only be suggestive but not diagnostic in case of Calvarial TB. ${ }^{9}$ CT scan and magnetic resonance imaging (MRI) are strongly recommended to assess extent of bone involvement, scalp swelling and the amount of intracranial extension. In our case, CT showed punched-out lytic lesion with subperiosteal abscess.

Demonstration of acid-fast bacilli (AFB) in the smears or biopsy specimen is diagnostic, but due to severe necrosis in some specimens' AFB may not be demonstrable. $^{10}$ In our patient, surgical debridement was done. On histopathology caseous necrosis was seen. Ziehl Neelsen (ZN) stain of the sample showed AFB, confirming the diagnosis of tuberculosis of this region.

Management of this condition include antitubercular therapy (ATT) and surgery. Surgical management by debridement and curettage wasthe sole modality of treatment which showed very good results before the start of $A \pi T .{ }^{2} A s$ in our case both ATT along with surgery were used for treatment with a good outcome, as the patient improved and was discharged home. Recent studies suggest that combination therapy (ATT with surgery) is far better as more areas of involved bone, will be the source of releasing tubercle bacilli, if surgery is delayed. ${ }^{10,11}$

Prognosis solely relies on the associated tuberculous lesion and local extent of the disease. Our case had a good prognosis as there was limited local invasion of the disease.

Conclusion

Calvarial TB can present in an elderly patient as a scalp swelling without a discharging sinus. Typical features on CT brain and FNAC with AFB staining help in the definitive diagnosis. Timely surgical intervention followed by initiation of anti-tubercular therapy are associated with good prognosis.

\section{References}

1. Datta SGS, Bhatnagar V, Pan S, Mehta R, Sharma C. Primary calvarial tuberculosis: a report of three cases. Br J Neurosurg. 2019; 33(2): 196-201. Doi: 10.1080/02688697.2017.1409879. 
2. Sridharan S, Balasubramanian D. Primary calvarial tuberculosis. Surg Neurol Int. 2017; 8: 126. Doi: 10.4103/sni.sni_495_16.

3. Ravikanth R, Mathew S, Selvam RP. Calvarial tuberculosis presenting as multiple osteolytic soft tissue lesions. CHRISMED J Health and Res. 2017; 4(4): 278-9. Doi: 10.4103/cjhr.cjhr_36_17.

4. Homagain S, Shrestha S, Sah S, Sedain G. Calvarial Tuberculosis: A Diagnostic Quandary: A Case Report. JNMA J Nepal Med Assoc. 2019; 57: 198-201. PMID: 31477963.

5. Bhoi SK, Naik S, Jha M, Pradhan N. Skull base and calvarial tuberculosis presenting as chronic meningitis. IJTB. 2020; 67(3): 411-3. Doi: 10.1016/j.ijtb.2020.01.011.

6. Awasthy $\mathrm{N}$, Chand $\mathrm{K}$, Singh A. Calvarial tuberculosis: Review of six cases. Ann Indian Acad Neurol. 2006; 9(4): 227-29. Doi: 10.4103/0972-2327.29205

7. Akpınar A, Erdoğan U, Koçyiğit M. Primary Calvarial Tuberculosis Presenting with Scalp Swelling and Lytic
Bone Lesion: A Case Report. Med Bull Haseki. 2018; 56(3): 240-3. Doi: 10.4274/haseki.3532.

8. Mackowiak PA, Yalamanchili M, Ada S, Bradstreet P. A 27-year-old man with soft-tissue swelling on the scalp and hypochondrium. Clin Infect Dis. 2002; 35(6): 763-4.

9. Choo Y-H, Seo Y, Choi J. Calvarial Tuberculosis Presenting with Skin Defect in an Elderly Patient. World Neurosurg. 2020; 140: 267-70. Doi: 10.1016/j.wneu.2020.05.076.

10. da Silva Martins AMS, Fanjul F, Rosselló AAC, Jaume MR. Calvarial tuberculosis: an unusual presentation of disseminated tuberculosis. BMJ Case Rep. 2019; 12(4): e226967. Doi: 10.1136/bcr-2018-226967.

11. Sant'Anna CC, Perinei PL, Pessôa AC, Goudouris E, Frota AC. Calvarial tuberculosis: two cases in children. Rev Inst Med Trop S Paulo. 2020; 62. Doi: 10.1590/s1678-9946202062068. 